\title{
Riccati equation and the problem of decoherence II: Symmetry and the solution of the Riccati equation
}

\author{
Bartłomiej Gardas ${ }^{1, \text { a) }}$ \\ Institute of Theoretical and Applied Informatics, Polish Academy of Sciences, \\ Battycka 5, 44-100 Gliwice, Poland
}

(Dated: 28 February 2010)

In this paper we revisit the problem of decoherence applying the block operator matrices analysis. Riccati algebraic equation associated with the Hamiltonian describing the process of decoherence is studied. We prove that if the environment responsible for decoherence is invariant with respect to the antylinear transformation then the antylinear operator solves Riccati equation in question. We also argue that this solution leads to neither linear nor antilinear operator similarity matrix. This fact deprives us the standard procedure for solving linear differential equation (e.g., Schrödinger equation). Furthermore, the explicit solution of the Riccati equation is found for the case where the environment operators commute with each other. We discuss the connection between our results and the standard description of decoherence (one that uses the Kraus representation). We show that reduced dynamics we obtain does not have the Kraus representation if the initial correlations between the system and its environment are present. However, for any initial state of the system (even when the correlations occur) reduced dynamics can be written in a manageable way.

PACS numbers: 03.65.Yz, 03.67.-a

\footnotetext{
a)Electronic mail: bartek.gardas@gmail.com
} 


\section{INTRODUCTION}

Recently, the connection between a problem of decoherence and the Riccati operator equation was established (for details see ${ }^{1}$ and Ref. therein). Moreover, it was shown that a wide class of a time-dependent quantum systems, precisely the ones that describe a qubit $Q$ interacting with the environment $E$ and defined by the following Hamiltonian

$$
H_{Q E}(t, \beta)=H_{Q}(t, \beta) \otimes \mathbb{I}_{E}+\mathbb{I}_{Q} \otimes H_{E}+f\left(\sigma_{3}\right) \otimes V
$$

where the Hamiltonian $H_{Q}(t, \beta)$ of the qubit alone is give by

$$
H_{Q}(t, \beta)=\beta \sigma_{3}+\alpha\left(\sigma_{1} \cos (\omega t)+\sigma_{2} \sin (\omega t)\right)
$$

where $\alpha, \beta \in \mathbb{R}$, can be effectively simplified to the time-independent problem. Namely the one governed by the Hamiltonian $H_{Q E}(0, \beta) \equiv H_{Q E}$. The connection between the reduced dynamics ${ }^{2}$ of those models can be summarized in the following formula

$$
\bar{\rho}_{t}=V_{t} \rho_{t}(\bar{\beta}) V_{t}^{\dagger}
$$

where $\bar{\beta}:=\beta-\omega / 2$ plays the role of effective parameter and $V_{t}:=\operatorname{diag}\left(e^{-i \omega t / 2}, e^{i \omega t / 2}\right)$ is the unitary (similarity) transformation. Here, $\bar{\rho}_{t}$ is the solution (reduced dynamics) of the system identified with the time-dependent Hamiltonian $H_{Q E}(t, \beta)$ and $\rho_{t}(\beta)$ representing the reduced dynamics of the model described by the Hamiltonian $H_{Q E}$. An explicit dependence $\bar{\rho}_{t}$ of $\beta$ was omitted. The meaning of the symbols we used in the equations (1) and (2) is usual. Furthermore, it was found that this time-independent problem can be solved in two ways. One could either resolve for $X$ the Riccati operator equation:

$$
\alpha X^{2}+X\left(H_{+}+\beta\right)-\left(H_{-}-\beta\right) X-\alpha=0
$$

where $H_{ \pm}:=H_{E} \pm V$, or solve the following Schrödinger equation (we work with the units $\hbar=1)$

$$
i \dot{\Psi}_{t}=H_{t} \Psi_{t}, \quad H_{t}=\left[\begin{array}{cc}
H_{E} & z_{t}^{*} V_{\beta} \\
z_{t} V_{\beta} & H_{E}
\end{array}\right]
$$


with initial condition $\Psi_{0} \equiv \Psi$. In Eq. (5) $\Psi_{t}=\left[\psi_{t}, \phi_{t}\right]^{T} \in \mathcal{H} \oplus \mathcal{H}, z_{t}=e^{-i 2 \alpha t}$ and $V_{\beta}=$ $V+\beta \mathbb{I}_{E}$. In the description above it was assumed that $\mathcal{H}$ is a separable Hilbert space (possibly infinite-dimensional) and $H_{E}$ and $V$ are the Hermitian operators acting on it. One could recall that in the current paper we assume that the Riccati equation $R_{H}[X]=0$, where

$$
R_{H}[X]=X B X+X A-C X-B^{\dagger}
$$

is associated with the following Hamiltonian

$$
H=\left[\begin{array}{cc}
A & B \\
B^{\dagger} & C
\end{array}\right] .
$$

In turn if the solution of the equation $R_{H}[X]=0$ exists, it may be used to diagonalize operator matrix $H$. Following equality holds true

$$
S_{X}^{-1} H S_{X}=\left[\begin{array}{cc}
A+B X & 0 \\
0 & A-(X B)^{\dagger}
\end{array}\right],
$$

where $S_{X}^{-1}$ stands for the inversed operator matrix to

$$
S_{X}=\left[\begin{array}{cc}
\mathbb{I}_{E} & -X^{\dagger} \\
X & \mathbb{I}_{E}
\end{array}\right]
$$

The Riccati Eq. (4) is associated with the Hamiltonian $H_{E Q}$. On the other hand the Riccati equation related to the Hamiltonian $H_{t}$ reads

$$
X\left(z_{t}^{*} V_{\beta}\right) X+X H_{E}-H_{E} X-z_{t} V_{\beta}=0 .
$$

One should keep in mind that the given operator $A$ acting on the space $\mathbb{C}^{2} \otimes \mathcal{H}$ may be thought of as a block operator matrix, since the following isomorphism holds $\mathbb{C}^{2} \otimes \mathcal{H}=\mathcal{H} \oplus \mathcal{H}$.

It is worth mentioning that in spite of the fact that connection between operator matrices $H_{t}$ and $H_{Q E}$ is well defined and the solution of the Eq. (10) can be easily obtained (indeed, it is given by $X_{t}=z_{t} \mathbb{I}_{E}$ ) the solution of the Eq. (4) is still missing. Please note that although $X_{t}$ is known it cannot be effectively used to resolve Eq. (5) because of its explicit time dependence. Nevertheless it allows us to diagonalize the Hamiltonian $H_{t}$, namely 


$$
S_{z_{t}}^{\dagger} H_{t} S_{z_{t}}=\left[\begin{array}{cc}
H_{+}+\beta \mathbb{I}_{E} & 0 \\
0 & H_{-}-\beta \mathbb{I}_{E}
\end{array}\right] \equiv H^{d}
$$

where the unitary matrix $S_{z_{t}}$ is defined as

$$
S_{z_{t}}=\frac{1}{\sqrt{2}}\left[\begin{array}{cc}
\mathbb{I}_{E} & -z_{t}^{*} \mathbb{I}_{E} \\
z_{t} \mathbb{I}_{E} & \mathbb{I}_{E}
\end{array}\right]=\frac{1}{\sqrt{2}}\left(\begin{array}{cc}
1 & -z_{t}^{*} \\
z_{t} & 1
\end{array}\right) \otimes \mathbb{I}_{E}
$$

We want to emphasize that the Eq. (10) and its slight modification

$$
z_{t}^{*} X V_{\beta} X+X H_{E}-H_{E} X-z_{t} V_{\beta}=0
$$

used by the author in previous manuscript on the subject ${ }^{1}$, are equivalent only if the solution is assumed to be a linear operator. This seems to be justified, especially if one expects $X$ to represent an observable. However, it does not need to be true. Thus in this manuscript we will not restrict the analysis to the linear operators only.

The purpose of this manuscript is two fold. Firstly, we show that if the environment is (i.e., the operators $H_{E}$ and $V$ are) invariant under antylinear transformation (Sec. II), then this antylinear operator is the solution of the Riccati equation (10). We also indicate that mentioned symmetry may be used to diagonalize the Hamiltonian related with Eq. (4), although it does not solve this equation. Next, in Sec. (III) we argue that the solution we obtained can not be applied to solve Eq. (5). The problem occurs because standard methods provided by the theory of the differential equations demand that the operator is not antylinear. The reduced dynamics of the system under consideration is given in Sec. (V).

Secondly, in Section (IV) we study the case when $\left[H_{E}, V\right]=0$ and we give the exact solution of the Riccati equation (4) for this situation. This is direct generalization of the recently found solution for the particular operator defining the spin-environment. Finally, in Sec. (VI) we compare our method with the standard approach based on the operator sum representation. We also discuss the possibility of obtaining an operator (or Kraus) sum representation in the case when the correlations between the system and its environment are present initially. 


\section{SYMMETRY AND THE SOLUTION TO THE RICCATI EQUATION}

Let $\tau_{1}$ and $\tau_{2}$ be an antilinear symmetry for $H_{E}$ and $V$, such that $\tau_{i}^{2}=\mathbb{I}_{E}$, (or $\left.\tau_{i}=\tau_{i}^{-1}\right)$ for $i=1,2$, respectively. By definition, it means that $\left[H_{E}, \tau_{1}\right]=0$ and $\left[V, \tau_{2}\right]=0$. Since the symmetry operator is an involution i.e., $\tau^{2}=\mathbb{I}_{E}$, thus for a given operator $A$ the condition $[\tau, A]=0$ is equivalent to the equality $\tau A \tau^{-1}=A$. Operators that fulfill last equality are invariant under the action of $\tau$ and are called $\tau$-symmetric. Furthermore, the statement that an operator $\tau$ acting on $\mathcal{H}$ is an antilinear has the following meaning

$$
\tau(a \psi+b \phi)=a^{*} \tau \psi+b^{*} \tau \phi,
$$

for every $\psi, \phi \in \mathcal{H}$ and $a, b \in \mathbb{C}$. We wish to emphasize that in the finite-dimensional case the existence of the aforementioned symmetry is ensured by the theorem of Ali Mostafazadeh ${ }^{3}$ which states that every diagonalazable pseudo-Hermitian (in particular the Hermitian) operator $H$ with the discrete spectrum has an antilinear and anti-hermitian symmetry $\tau$. Moreover, this symmetry is an involution, i.e., $\tau^{2}=\mathbb{I}_{E}$, if $H$ is Hermitian operator, i.e., $H=H^{\dagger}$. The proof of this theorem provides the explicit construction of $\tau$. Let us additionally assume that $\tau_{1}=\tau_{2}:=\tau$, which means that the operators $H_{E}$ and $V$ posses a common symmetry $\tau$.

If one allow $X$ to be an antilinear operator, then equation (10), can be rewritten in the following form

$$
R_{H_{t}}[X]=z_{t}\left(X V_{\beta} X-V_{\beta}\right)+\left[X, H_{E}\right] .
$$

From this equation we can readily see that $R[\tau]=0$, i.e., the symmetry generator $\tau$ of the $E$ system is the solution we were seeking for. In particular, if the Hamiltonians $H_{E}$ and $V$ are (or roughly speaking the $E$ system is) $P T$-symmetric (or $T$-symmetrix) where $P$ and $T$ stand for the parity and the time-reversal operators, respectively, then $X=P T(X=T)$, i.e., the $P T(T)$ operator solves Eq. (10). This is very interesting and rather unexpected result that the famous $P T(T)$ symmetry is the solution of the Riccati equation we study.

For instance it can be easily proven that if both $H_{E}$ and $V$ are symmetric operators, that is to say $H_{E}^{T}=H_{E}$ and $V^{T}=V$, where by " $T$ " we denoted the operation of transposition,

then it implies that $R_{H_{t}}[K]=0$, where $K$ is complex conjugate operator (see below). In order to do that let us define 


$$
K \psi=\psi^{*}, \quad \psi \in \mathcal{H},
$$

where by "*" stands for the standard complex conjugation operation. The operator above possesses the following properties:

a) $K=K^{\dagger}$ (i.e., it is Hermitian operator),

b) $K K^{\dagger}=\mathbb{I}_{E}$ (i.e., it is unitary operator),

c) $K^{2}=\mathbb{I}_{E}$ (i.e., it is an involution).

Moreover, $K$ is antilinear operator. The listed properties follow immediately from the definition (16). Note that for any Hermitian operator (matrix) $A$ the condition $A^{T}=A$ means that $A$ is $K$-symmetric, i.e., $[A, K]=0$. To see this observe that $K$ transforms any operator $A$ in accord with the following rule:

$$
K A K^{\dagger}=A^{*}
$$

In addition, if one assumes that $A$ is Hermitian, i.e., $A=A^{\dagger}$ and uses properties $a$ ) then from the equation above we have

$$
K A K=A^{T}
$$

where we used the fact that $A^{\dagger}=\left(A^{T}\right)^{*}$. Clearly, for the symmetric matrix $K A K=A$ or $[A, K]=0$. Other way to see that $R_{H_{t}}[K]=0$ is to rewrite $R[K]$ as

$$
R_{H_{t}}[K]=z_{t}\left(V_{\beta}^{T}-V_{\beta}\right)+\left(H_{E}^{T}-H_{E}\right) K
$$

Evidently, for the symmetric operators the right side of the Eq. (19) vanishes.

At the end of this section we show how to diagonalize the operator $H_{Q E}$ using the symmetry $\tau$. Firstly, note that if one introduces the unitary operator $U$ in a way that

$$
U=\frac{1}{\sqrt{2}}\left[\begin{array}{cc}
\mathbb{I}_{E} & i \mathbb{I}_{E} \\
i \mathbb{I}_{E} & \mathbb{I}_{E}
\end{array}\right]=\frac{1}{\sqrt{2}}\left(\begin{array}{ll}
1 & i \\
i & 1
\end{array}\right) \otimes \mathbb{I}_{E}
$$


then $U^{\dagger} H_{Q E} U=\bar{H}$ and

$$
\bar{H}=\left[\begin{array}{cc}
H_{R} & V_{\alpha \beta} \\
V_{\alpha \beta}^{\dagger} & H_{R}
\end{array}\right]
$$

where $V_{\alpha \beta}:=\alpha \mathbb{I}_{E}-i V_{\beta}$. Since $\tau V_{\alpha \beta} \tau=V_{\alpha \beta}^{\dagger}$, thus from Eq. (21) we see that $R_{\bar{H}}[\tau]=0$, i.e., the symmetry $\tau$ is the solution of the Riccati equation associated with the Hamiltonian (21). Therefore, the matrix $\bar{S}_{\tau}:=U S_{\tau}$ diagonalizes the Hamiltonian $H_{Q E}$. To be specific, the following equation holds true

$$
\bar{S}_{\tau}^{\dagger} H_{Q E} \bar{S}_{\tau}=\left[\begin{array}{cc}
H_{R}+V_{\alpha \beta} \tau & 0_{E} \\
0_{E} & H_{R}-V_{\alpha \beta}^{\dagger} \tau
\end{array}\right]
$$

Keep in mind that the operator $V_{\alpha \beta}$ is not Hermitian, while the operator $V_{\alpha \beta} \tau$ is. Interestingly, we diagonalized the block operator matrix $H_{Q E}$ without directly resolving the Riccati algebraic equation associated with it. One may ask if this is possible in general. To be specific if there exists a matrix $T$ such that it transforms a given operator matrix $A$ to another one $\bar{A}$ viz $\bar{A}=T^{-1} A T$ and the Riccati equation $R_{\bar{A}}[X]=0$ related to $\bar{A}$ has the solution $X_{\bar{A}}$ that is easy to be found. If that would be the case, than one may construct similarity operator matrix, namely $S_{X_{\bar{A}}} T$ that diagonalizes $A$. We saw that if $A=H_{Q E}$, then $T=U$ (Eq. (20)) and $\bar{A}=\bar{H}$ (Eq. (21)), thus the procedure is working. Therefore, this strategy is worth to be studied. However, we will not focus on this subject herein.

\section{THE PROBLEM WITH THE SCHRÖDINGER EQUATION}

The results of the paper ${ }^{1}$ and the last section show that $R_{H_{t}}\left[z_{t}\right]=0$ and $R_{H_{t}}[\tau]$, where $R_{H_{t}}[X]$ stands for the left side of the Eq. (10). Note that, the second solution $(X=\tau)$ we obtained, in contrast to the first one $\left(X_{t}=z_{t}\right)$ is time independent. Therefore, one may think that the second solution has the advantage because it allows one to construct the operator matrix $S_{\tau}$ that diagonalizes Hamiltonian (5) and do not depend on time. Indeed this is the case however one serious drawback arises. To see this clearly, let us give an explicit form of the matrix $S_{\tau}$. In agreement with Eq. (9) it takes the form (compare this with Eq. (12)) 


$$
S_{\tau}=\frac{1}{\sqrt{2}}\left[\begin{array}{cc}
\mathbb{I}_{E} & -\tau \\
\tau & \mathbb{I}_{E}
\end{array}\right]
$$

Since the symmetry $\tau$ is an involution operator $\left(\tau^{2}=\mathbb{I}_{E}\right)$, thus $S_{\tau}$ is the unitary matrix $\left(S_{\tau} S_{\tau}^{\dagger}=\mathbb{I}_{E}\right)$. We see that the similarity transformation (23) is neither linear nor antilinear operator. In particular, one may easily verify that $S_{\tau} i=i S_{\tau}^{\dagger}$. All this difficulties are direct consequence of the fact that the operator $\tau$ is antilinear. As a result, we cannot use the standard procedure that allows us to solve linear, differential equation to resolve the Schrödinger equation $i\left|\dot{\Psi}_{t}\right\rangle=H_{t}\left|\Psi_{t}\right\rangle$. Indeed, because of the presence of the factor $i$ on the left side of the Schrödinger equation we cannot apply the $\left|\Phi_{t}\right\rangle=S_{\tau}\left|\Psi_{t}\right\rangle$ transformation to reduce it to the following form $i\left|\dot{\Phi}_{t}\right\rangle=H_{t}^{d}\left|\Phi_{t}\right\rangle$, where $H_{t}^{d}$ stands for the diagonal form of $H_{t}$. Another technique is needed to resolve this difficulties. Unfortunately, at the present time there is none. Of course, the same problems occur when one tries to resolve the equation $i\left|\dot{\Psi}_{t}\right\rangle=H_{Q E}\left|\Psi_{t}\right\rangle$ using the Eq. (22).

Notice that according to procedure explained in the paper ${ }^{1}$ the $H_{t}$ given by Eq. (5) has the following block-diagonalization (compare with Eq. (11))

$$
S_{\tau}^{\dagger} H_{t} S_{\tau}=\left[\begin{array}{cc}
H_{E}+z_{t}^{*} V_{\beta} \tau & 0 \\
0 & H_{E}-z_{t} V_{\beta} \tau
\end{array}\right] \equiv H_{t}^{d} .
$$

Comparing equations (11) and (24) one can learn that the solution $X_{t}=z_{t}$ leads to the timeindependent diagonal form $H^{d}$ of the operator $H_{t}$, but the transformation matrix $S_{t}$ does depend on time. Opposite situation takes place in the case of the second solution $X=\tau$, i.e., diagonal form $H_{t}^{d}$ is time-dependent and transformation matrix $S_{\tau}$ do not depend on time.

\section{SOLUTION OF THE RICCATI EQUATION.}

Recently, the solution of the model specified by the Eq. (1) was given in the case when $\mathcal{H}=\bigotimes_{n=1}^{N} \mathbb{C}^{2}$ and the operator $H_{E}, V$ are defined as (for detailed discussion $\operatorname{see}^{4}$ )

$$
H_{E}=\sum_{n=1}^{N} \omega_{n} \sigma_{3}(n), \quad V=\sum_{n=1}^{N} g_{n} \sigma_{3}(n)
$$


where $\omega_{n}$ and $g_{n}$ are certain constants defining the frequencies and the coupling constant of the spin-bath, respectively. For every $n \leq N$ the operator $\sigma_{3}(n)$ is understood as $\sigma_{3}(n)=$ $I_{2} \otimes \ldots \otimes \sigma_{3} \otimes \ldots \otimes I_{2}$, where $I_{2}$ is a $2 \times 2$ identity matrix and $\sigma_{3}$ is the usual Pauli matrix. Note that the operators above commute, i.e., $\left[H_{E}, V\right]=0$. We will extend results obtained in paper $^{4}$ to the arbitrary operators that commute with each other.

If one assumes that the operators $H_{E}$ and $V$ commute, then they have common set of eigenvectors. Henceforward, we will assume that the eigenvalues of $H_{E}$ and $V$ are all discrete and not degenerated. Furthermore, the spectrum of a given operator $A$ will be denoted by $\sigma(A)$. As a summary, one can write the following eigenvalue problems for $H_{E}$ and $V$ :

$$
H_{E}\left|\phi_{n}\right\rangle=E_{n}\left|\phi_{n}\right\rangle, \quad V\left|\phi_{n}\right\rangle=V_{n}\left|\phi_{n}\right\rangle
$$

where $E_{n} \in \sigma\left(H_{E}\right)$ and $V_{n} \in \sigma(V)$. Here, the index $n$ goes through the set of all integer numbers or through each subset of it. We wish to emphasize that the assumption of the discreetness and no degeneration of the spectrum of the operators in question is not crucial in our analysis. In fact, it can by easily overcome (however, we will not address this technical issue in the current manuscript).

Note, the solution $X$ of the Eq. (4) is a function of the operators $H_{ \pm}$defined in the Eq. (4). Since the operators $H_{E}$ and $V$ commute with each other, so are the operators $H_{ \pm}$. Therefore, $\left[X, H_{ \pm}\right]=0$ and the Riccati equation (4) can be simplified to the following form

$$
\alpha X^{2}+2 V_{\beta} X-\alpha=0 \text {. }
$$

One can observe that if $\alpha=0$ then $X_{0}=0_{E}$ is the solution of the Eq. (27). No wonder since in this case matrix $H_{Q E}$ is already in the diagonal form. Yet, the operator $X_{0}$ may not be the only solution of the equation $R_{H_{Q E}}[X]=0$.

In order to obtain the solution for the case where $\alpha \neq 0$ we will apply the spectral theorem for Hermitian operators. From the Eq. (27) one can readily see that $X=f\left(V_{\beta}\right)$, where the function $f$ takes the form

$$
f(\lambda)=\frac{-\lambda+\sqrt{\lambda^{2}+\alpha^{2}}}{\alpha}, \quad \lambda \in \sigma\left(V_{\beta}\right)
$$

and $\sigma\left(V_{\beta}\right)=\sigma\left(V+\beta \mathbb{I}_{E}\right)$. One may also write the operator $f(V)$ in the equivalent way: 


$$
f(V)=\sum_{\lambda \in \sigma\left(V_{\beta}\right)} f(\lambda)|\lambda\rangle\langle\lambda| .
$$

Thus, in view of the Eq. (26) and (29) the $X$ takes the final form

$$
X=\sum_{n} x_{n}\left|\phi_{n}\right\rangle\left\langle\phi_{n}\right|
$$

where abbreviation $x_{n} \equiv f\left(V_{n}+\beta\right)$ was introduced. Note that $x_{n}$ are the eigenvalues of the operator $X$, that is $X\left|\phi_{n}\right\rangle=x_{n}\left|\phi_{n}\right\rangle$. The operator above is Hermitian, i.e., $X=X^{\dagger}$. Since for every parameter $\alpha$ and $\beta$ the function $f$ specified by the Eq. (28) takes positive values, thus the eigenvalues $x_{n}$ are positive. Therefore, the solution we obtained in the Eq. (30) is positively defined operator. Moreover, as was pointed out in ${ }^{4}$ there exists at least one more solution of the Eq. (27), in the case where $H_{E}$ and $V$ were chosen to be the ones that describe the spin-bath (see Eq. (25)). This situation also occurs when the operators $H_{E}$ and $V$ have more general form, then the second solution is given by

$$
\bar{f}(\lambda)=\frac{-\lambda-\sqrt{\lambda^{2}+\alpha^{2}}}{\alpha}, \quad \lambda \in \sigma\left(V_{\beta}\right) .
$$

It can be easily verified that this function determins the negatively defined operator, that is to say

$$
\bar{X}=\sum_{n} \bar{x}_{n}\left|\phi_{n}\right\rangle\left\langle\phi_{n}\right|
$$

and $\bar{x}_{n}:=\bar{f}\left(V_{n}+\beta\right)$. Observe that $\bar{X}=\bar{X}^{\dagger}$, i.e., this operator is also Hermitian. Note also that $f(\lambda) \bar{f}(\lambda)=-1$, for $\lambda \in \sigma\left(V_{\beta}\right)$. This may be verified directly, or may also be thought of as a consequence of the Viet'a formulas, if one thinks of $f$ and $\bar{f}$ as the solution of the quadratic equation: $\alpha x^{2}+\lambda x-\alpha=0$, for $\lambda \in \sigma\left(V_{\beta}\right)$. We want to emphasize however, that the Riccati Eq. (27) is not a simple quadratic polynomial, even though it may appear so. As a result there might exist other solutions of this equation that are not specified by the well-known formula for roots of the quadratic equation.

At this point natural questions may by asked, for instance which operator, $X$ or $\bar{X}$ (or any other, if it exist) should by used to diagonalize the block operator matrix $H_{Q E}$ ? What difference (if any) does it make? Of course, each solution of the Riccati equation will diagonalize the operator matrix with which it is associated. Nevertheless, in certain cases 
it may by convenient to chose one solution instead of the other. For example by studying limiting cases like $\alpha \rightarrow 0$. Indeed, if $\beta \neq 0$, then we find that (recall that $f \sim 1 / \bar{f}$ )

$$
\lim _{\alpha \rightarrow 0} f=0, \quad \lim _{\alpha \rightarrow 0} \bar{f}=-\infty .
$$

Therefore, $X \rightarrow 0_{E}$ while $\bar{X} \rightarrow-\infty$ as $\alpha$ goes to 0 . This means that the first solution is a continuous function of the parameter $\alpha$, including the $\alpha=0$ value even though the second operator, i.e., $\bar{X}$ does not exist in that point at all. As a result, if in the process of analysis one decides to use the second solution $\bar{X}$ then one might meet serious problems taking the limits $\alpha \rightarrow 0$. Furthermore, as was mentioned earlier the first solution is a positively defined operator, thus it is more suitable to manage. Henceforward, we will restrict further analysis to the operator $X$ given by the Eq. (30) only.

\section{THE EXACT REDUCED DYNAMICS}

We now use the solution above to construct the exact reduced dynamics of the model described by $H_{Q E}$. Obtaining the exact reduced dynamics of the model under consideration, namely the one defined by the Eq. (1), can be easily accomplished using the Eq. (3), as we mentioned earlier. We begin with the construction of the evolution operator $U_{t}$ generated by the Hamiltonian $H_{Q E}$. Foremost, let us recall that the evolution operator $U_{t}$ may be computed by applying the following formula

$$
U_{t}=S_{X} \exp \left(-i H_{d} t\right) S_{X}^{-1}
$$

where $H_{d}$ stands for the diagonal form of the Hamiltonian $H_{Q E}$ and in agreement with Eq. (8) it takes the form

$$
H_{d}=\left[\begin{array}{cc}
H_{+}+\alpha X & 0_{E} \\
0_{E} & H_{-}-\alpha X
\end{array}\right],
$$

where $X$ is given by (30). Next let us observe that the inverse operator $S_{X}^{-1}$ is

$$
\begin{aligned}
S_{X}^{-1} & =\left[\begin{array}{cc}
g(X) & 0_{E} \\
0_{E} & g(X)
\end{array}\right] S_{X}^{\dagger} \\
& \equiv G(X) S_{X}^{\dagger},
\end{aligned}
$$


where $g(X)$ is the function of $X$ and it is given by

$$
g(\lambda)=\frac{1}{1+\lambda^{2}}, \quad \lambda \in \sigma(X)
$$

Note that $[X, V]=0$, thus $X$ and $V$ have the same eigenstate, namely $\left|\phi_{n}\right\rangle$. Since $X=f\left(V_{\beta}\right)$,

the eigenvalues $x_{n}$ of $X$ are given by $x_{n}=f\left(V_{n}+\beta\right)$. Obviously, $\sigma(X)=f\left(\sigma\left(V_{\beta}\right)\right)$. The same arguments lead to the conclusion that $g(X)\left|\phi_{n}\right\rangle=(g \circ f)\left(E_{n}+\beta\right)\left|\phi_{n}\right\rangle$, this implies that $\sigma(g(X))=(g \circ f)\left(\sigma\left(V_{\beta}\right)\right)$. Using the Eqs. (34) - (36) one may finally write the form of the evolution operator of the total system, it reads

$$
U_{t}=G(X)\left[\begin{array}{cc}
U_{t}^{+}+X^{2} U_{t}^{-} & \left(U_{t}^{+}-U_{t}^{-}\right) X \\
\left(U_{t}^{+}-U_{t}^{-}\right) X & U_{t}^{-}+X^{2} U_{t}^{+}
\end{array}\right],
$$

where $U_{t}^{ \pm}:=\exp \left(-i\left(H_{ \pm} \pm \alpha X\right) t\right)$. Equation (38) represents the evolution operator of the total system $Q+E$ and it can be easily applied to any (not only to the factorable ones) initial state $\rho_{Q E}$ of that system, since it is written in $2 \times 2$ block operator matrix. At this point reduced dynamics $\rho_{Q}(t)$ may by obtained, it has the following form

$$
\rho_{Q}(t)=\operatorname{Tr}_{E}\left(U_{t} \rho_{Q E} U_{t}^{\dagger}\right)
$$

where $\operatorname{Tr}_{E}$ is the partial trace operation. Note that in general case this may not be easy to accomplish, even though the Eq. (39) might indicate so. The reason for that is that to compute partial trace $\operatorname{Tr}_{E}$ one needs to write operator $\rho_{Q E}(t)$ in the $2 \times 2$ block operator form.

\section{KRAUS REPRESENTATION}

It is interesting to see how the results of the previous section are related to the standard description of the completely positive map via so called Kraus sum representation:

$$
\rho_{Q}(t)=\sum_{\mu} K_{\mu}(t) \rho_{Q} K_{\mu}(t)^{\dagger}
$$

where the Kraus matrices $K_{\mu}(t)$ satisfy following completeness relation $\sum_{\mu} K_{\mu}(t) K_{\mu}(t)^{\dagger}=$ $\mathbb{I}_{Q}$. It is well established that it is possible to derive the Eq. (40) from the Eq. (39) assuming that no correlations between the system and its environment are present initially ${ }^{2,5}$. The 
generalization to the case when initial state is not factorable is also possible ${ }^{6,7}$ (see discussion below). Nevertheless, in practice finding the Kraus matrices is impossible in most cases. We will show how to construct those matrices for the system we study. To accomplish this goal let us rewrite $S_{X}$ in the following manner

$$
\begin{aligned}
S_{X} & =\sum_{n}\left(\begin{array}{cc}
1 & -x_{n} \\
x_{n} & 1
\end{array}\right) \otimes\left|\phi_{n}\right\rangle\left\langle\phi_{n}\right| \\
& \equiv \sum_{n} F_{n} \otimes\left|\phi_{n}\right\rangle\left\langle\phi_{n}\right| .
\end{aligned}
$$

We also used resolution of the identity $\mathbb{I}_{E}$ in the $\left|\phi_{n}\right\rangle$ basis, that is to say $\mathbb{I}_{E}=\sum_{n}\left|\phi_{n}\right\rangle\left\langle\phi_{n}\right|$. Note the inverse operator $S_{X}^{-1}$ can by written in a similar fashion, namely

$$
S_{X}^{-1}=\sum_{n} F_{n}^{-1} \otimes\left|\phi_{n}\right\rangle\left\langle\phi_{n}\right|
$$

where $F_{n}^{-1}$ is the inverse matrix of $F_{n}$. Since we have $\operatorname{det}\left(F_{n}\right)=1+x_{n}^{2}>0$ it always exists. Due to the fact that $F_{n}^{-1}=F_{n}^{\dagger} / \operatorname{det}\left(F_{n}\right)$ holds we can rescale $F_{n}$ namely $F_{n} \rightarrow 1 / \sqrt{\operatorname{det}\left(F_{n}\right)} F_{n}$ so it becomes the unitary operator. Furthermore, the Hamiltonian (35) may be rewritten as

$$
\begin{aligned}
H_{d} & =\sum_{n}\left(\begin{array}{cc}
h_{n}^{+} & 0 \\
0 & h_{n}^{-}
\end{array}\right) \otimes\left|\phi_{n}\right\rangle\left\langle\phi_{n}\right| \\
& \equiv \sum_{n} H_{n}^{d} \otimes\left|\phi_{n}\right\rangle\left\langle\phi_{n}\right|,
\end{aligned}
$$

where $h_{n}^{ \pm}:=\left(E_{n}^{ \pm} \pm \beta\right) \pm \alpha x_{n}$ and $E_{n}^{ \pm}:=E_{n} \pm V_{n}$ are the eigenvalues of $H_{ \pm}$(note $\sigma\left(H_{ \pm}\right)=$ $\left.\left.\sigma\left(H_{E} \pm V\right)\right)\right)$. Combining Eq. (34) and Eqs. (41) - (43) we obtain

$$
U_{t}=\sum_{n} U_{n}(t) \otimes\left|\phi_{n}\right\rangle\left\langle\phi_{n}\right|,
$$

with the unitary matrices $U_{n}(t)=\exp \left(-i H_{n} t\right)$ and $H_{n}=F_{n}^{-1} H_{n}^{d} F_{n}$. One can see from the form of the evolution operator above and Eq. (39) that we finally have

$$
\begin{aligned}
\rho_{Q}(t) & =\sum_{n} \rho_{n} U_{n}(t) \rho_{Q} U_{n}(t)^{\dagger} \\
& \equiv \sum_{n} K_{n}(t) \rho_{Q} K_{n}(t)^{\dagger} .
\end{aligned}
$$


In the Eq. (45) $\rho_{n}:=\left\langle\phi_{n}\left|\rho_{E}\right| \phi_{n}\right\rangle$ and the Kraus matrices are defined as $K_{n}(t):=\sqrt{\rho_{n}} U_{n}(t)$. Therefore, the operator sum representation of the model we study is found.

\section{A. Connection with the Riccati diagonalization}

Interestingly, the explicit form of the matrix $H_{n}$ reads

$$
H_{n}=\left(\begin{array}{cc}
E_{n}^{+}+\beta & \alpha \\
\alpha & E_{n}^{-}-\beta
\end{array}\right),
$$

and it does not depend on the eigenvalues $x_{n}$ of the operator $X$. Therefore, one may draw the conclusion that the Kraus matrices obtained in Eq. (45) do not depend on $x_{n}$. To solve this puzzle, notice first that in order to compute the Krause matrices one needs to determine the "evolution" operator $U_{n}(t)$. The later requires diagonalization of its "generator" $H_{n}$. Because $H_{n}=F_{n}^{-1} H_{n}^{d} F_{n}$ the dependence of the Kraus matrices on the eigenvalues $x_{n}$ is "hidden" in a way, in the similarity matrix $F_{n}$. It is important to realize that the diagonalization procedure $H_{n} \stackrel{F_{n}}{\longrightarrow} H_{n}^{d}$ differs from the standard diagonalization routine, which is based on the eigenvalue problem for the operator $H_{n}$. This new kind of algorithm is called Riccati diagonalization and was recently introduced in $^{8}$. One may see that it arises naturally in our analysis. Let us also recall that the similarity matrix $F_{n}$ is composed of the $n$th eigenvalue $x_{n}$ of the operator $X$, which is the solution of the Riccati Eq. (27), while the eigenvalues of the matrix $H_{n}$ are the solution of the following characteristic equation associated with $H_{n}$ :

$$
\lambda^{2}-\lambda \operatorname{Tr}\left(H_{n}\right)+\operatorname{det}\left(H_{n}\right)=0,
$$

We have already found its solution indirectly in the Eq. (43). The roots of this equation are given by $\lambda_{n}^{ \pm}=h_{n}^{ \pm}$. The corresponding eigenvector (not normalized) reads

$$
\boldsymbol{\lambda}_{n}^{+}=\left(\begin{array}{c}
-\bar{x}_{n} \\
1
\end{array}\right), \quad \boldsymbol{\lambda}_{n}^{-}=\left(\begin{array}{c}
-x_{n} \\
1
\end{array}\right) .
$$

Therefore, the similarity (not unitary) matrix $G_{n}=\left(\boldsymbol{\lambda}_{n}^{+}, \boldsymbol{\lambda}_{n}^{-}\right)$the one that also diagonalizes $H_{n}$ takes the form 


$$
G_{n}=\left(\begin{array}{cc}
-\bar{x}_{n} & -x_{n} \\
1 & 1
\end{array}\right) .
$$

The connection between similarity matrices $F_{n}$ and $G_{n}$ is following. If $F_{n} \equiv\left(\boldsymbol{\xi}_{n}^{1}, \boldsymbol{\xi}_{n}^{2}\right)$, then $\boldsymbol{\xi}_{n}^{1}=x_{n} \cdot \boldsymbol{\lambda}_{n}^{+}$and $\boldsymbol{\xi}_{n}^{2}=\boldsymbol{\lambda}_{n}^{-}$, since $x_{n} \bar{x}_{n}=-1$. Therefore the matrix $F_{n}$ is also composed of the eigenvectors of $H_{n}$, yet the matrices $G_{n}$ and $F_{n}$ are not similar, i.e., the invertible matrix $P_{n}$ such that $G_{n}=P_{n} F_{n} P_{n}^{-1}$ does not exist. It immediately follows from the fact that $\operatorname{Tr}\left(G_{n}\right) \neq \operatorname{Tr}\left(F_{n}\right)$ as well as $\operatorname{det}\left(G_{n}\right) \neq \operatorname{det}\left(F_{n}\right)$. Since the similarity transformation preserves the trace and determinant, thus the matrices $G_{n}$ and $F_{n}$ can not be similar. From the consideration above one may easily grasp the main difference between the two methods. In the standard approach one needs to determine the eigenvalues $\lambda_{n}^{ \pm}$of $H_{n}$ as was mentioned above (i.e., one need to solve Eq. (47)). On the other hand to use the Riccati diagonalization schema to our advantage we need to find $x_{n}$ (i.e., resolve the Riccati Eq. (27)). Note that in the case of the ordinary matrix, the Eq. (27) becomes the quadratic equation, but it differs from the characteristic Eq. (47). In our model the relation between $\lambda_{n}^{ \pm}$and $x_{n}$ may be summarized as

$$
\lambda_{n}^{ \pm}=\left(E_{n}^{ \pm} \pm \beta\right) \pm \alpha x_{n} .
$$

\section{B. Initial correlation}

Let us notice that if the initial correlations between system of interest and its environment are present, i.e., $\rho_{Q E}$ takes the form

$$
\rho_{Q E}=\sum_{i j} \gamma_{i j} \rho_{Q}^{i} \otimes \rho_{E}^{j},
$$

for some not factorable complex number $\gamma_{i j}$, namely $\gamma_{i j} \neq \gamma_{1}^{i} \gamma_{2}^{j}$, then reduced dynamics (39) cannot be written in the form (40). This not so obvious, since there are cases in which, even though initial correlations are present the reduced dynamics can still be written in the Kraus form ${ }^{9}$.

Yet, for a finite dimensional environment there exists a simple criterion ${ }^{7}$. It allows one to verify when the given state $\rho_{Q}(t)$ possesses the operator sum representation (40) if initial correlations are present. The necessary and sufficient condition for the later to holds true 
for any initially correlated state is that the joint dynamics has to be locally unitary, i.e., the evolution operator $U_{t}$ for the total system needs to be of the form

$$
U_{t}=U_{Q}(t) \otimes U_{E}(t)
$$

The operator $U_{Q}(t)\left(U_{E}(t)\right)$ describes the evolution of the system $Q(E)$ alone. Observe that if the evolution of the total system is not locally unitary that this not necessary need to implies that $\rho_{Q}(t)$ does not posses Kraus representation for particular initially correlated state $\rho_{Q}$. From the Eq. (44) one can readily see that the evolution operator (38) does not have the form (52) as one may expected. Note that from (51) and Eq. (44) we obtain

$$
\rho_{Q}(t)=\sum_{n} \sum_{i j} \varepsilon_{i j}^{n} U_{n}(t) \rho_{Q}^{i} U_{n}(t)^{\dagger}
$$

where $\varepsilon_{i j}^{n}=\gamma_{i j}\left\langle\phi_{n}\left|\rho_{E}^{j}\right| \phi_{n}\right\rangle$. Therefore, even if in this general case the reduced dynamics can not be written in the operator sum representation, one can still describe the evolution of the system in a manageable way.

\section{SUMMARY}

In this paper we investigated the Riccati algebraic equation associated with the Hamiltonian defining the process of decoherence in the case of one qubit. It was shown that if the environment is $\tau$-symmetric, where $\tau$ is antilinear involution, then $\tau$ is the solution of the Riccati equation under consideration. We indicated that even though the solution of the Riccati equation has been found it can not be applied to obtain the reduced dynamics, due to the problem with standard procedure allowing one to solve Schrödinger equation. We wish to emphasize that this result does not contradict with the previous paper ${ }^{1}$, where we claim that the solution of the Riccati equation enables one to rewrite the evolution operator generated by the Hamiltonian $H_{Q E}$ as $2 \times 2$ block operator matrix. Of course, the reason that problems occur is that the solution of the Riccati Eq. is antilinear.

Furthermore, we provided a full resolution of the problems introduced in ${ }^{1}$ for the case when operators defining the environment commute with each other (regardless of the existence of any symmetry in the system). We also derived the operator sum representation for that model assuming no correlations between the systems are present initially. Moreover, 
we also showed how to obtain the solution if the initial state of the total system in not factorable. This result is a direct generalization of the system discussed in ${ }^{4}$. We also indicated that the recently derived schema of so called Riccati diagonalization arises naturally in the model we considered.

\section{ACKNOWLEDGMENTS}

We acknowledge the financial support by the Polish Ministry of Science and Higher Education under the grant number N N519 442339. The author would like to thank Jarosław Adam Miszczak for helpful comments and suggestions.

\section{REFERENCES}

${ }^{1}$ B. Gardas, J. Math. Phys. 51, 062103 (2010).

${ }^{2}$ R. Alicki and K. Lendi, Quantum dynamical semigroups and applications, Lecture notes in physics (Springer-Verlag (Berlin, New York), 1987).

${ }^{3}$ A. Mostafazadeh, J. Math. Phys. 43, 3944 (2002).

${ }^{4}$ B. Gardas, (2010), e-print, arXiv:1005.5182v1.

${ }^{5}$ I. Bengtsson and K. Życzkowski, Geometry of Quantum States: An Introduction to Quantum Entanglement (Cambridge University Press, Cambridge, U.K., 2006).

${ }^{6}$ P. Štelmachovič and V. Bužek, Phys. Rev. A 64, 062106 (2001); Phys. Rev. A 67 (2003).

${ }^{7}$ H. Hayashi, G. Kimura, and Y. Ota, Phys. Rev. A 67, 062109 (2003).

${ }^{8}$ K. Fujii and H. Oike, "Riccati diagonalization of hermitian matrices," (2010), to appear in Int. J. Geom. Meth. Mod. Phys., arXiv:1004.1207v2.

${ }^{9}$ D. M. Tong et al., Laser Physics 16, 1512 (2006). 